\title{
SEARCH AND STUDY OF EXTENSIVE AIR SHOWER EVENTS WITH THE TUS SPACE EXPERIMENT
}

\author{
Maria Lavrova ${ }^{1, *}$ for Lomonosov-UHECR/TLE Collaboration \\ ${ }^{1}$ Joint Institute for Nuclear Research
}

\begin{abstract}
The TUS experiment is aimed to study the energy spectrum and arrival direction of Ultra High Energy Cosmic Rays at $\mathrm{E} \sim 100 \mathrm{EeV}$ from the space orbit by measuring the fluorescence yield of the Extensive Atmospheric Shower in the atmosphere. It is the first orbital telescope designed for such measurements and is taking data since May 19, 2016. The TUS apparatus structure, methods of UHECR on-line selection and off-line data analysis are described. A few UHECR EAS candidates have been found. Preliminary results of their studying are presented.
\end{abstract}

\section{Introduction}

The measurements of the Cosmic Ray (CR) energy spectrum, composition and arrival directions in a wide energy interval are an important part of modern particle physics and astrophysics. The TUS project's goal is the experimental study of Ultra High Energy Cosmic Rays (UHECR) at energies about $10^{20} \mathrm{eV}$. The fluorescent and Cherenkov radiation of the Extensive Air Showers (EAS) generated by UHECR particles should be detected in the Earth's atmosphere on the night side of the orbit at altitudes $400-500 \mathrm{~km}$. An important advantage of the space detector is the possibility of taking data from different arrival directions of the sky with the same apparatus and with the same systematic uncertainties. With such data the existing difference between the results of the Auger detector in the southern hemisphere and the TA detector in the northern hemisphere may be understood $[1,2]$. TUS is the first attempt to detect UHECRs from space. TUS is a pathfinder for the next, larger UHECR fluorescence detectors KLYPVE [3] and JEM-EUSO [4].

\section{The TUS detector construction}

The TUS detector on board the Lomonosov satellite was launched into orbit from the newly built Cosmodrome Vostochny on April 28, 2016 (Fig. 1 right). The "Lomonosov" satellite has a solar-synchronized orbit with an inclination of $\sim 97^{\circ}$, a period of circulation $\sim 94 \mathrm{~min}$, and heights 470-500 km. TUS began measurements in space on May 19, 2016. The first months of the TUS operation in orbit were devoted to the apparatus functionality measurements. After flight tests and tuning of TUS hardware and software on the satellite, regular data has been received since the end of August 2016. During regular operation, the detector measures the UV background level and adjusts the HV

\footnotetext{
*e-mail: lavrova@jinr.ru
}
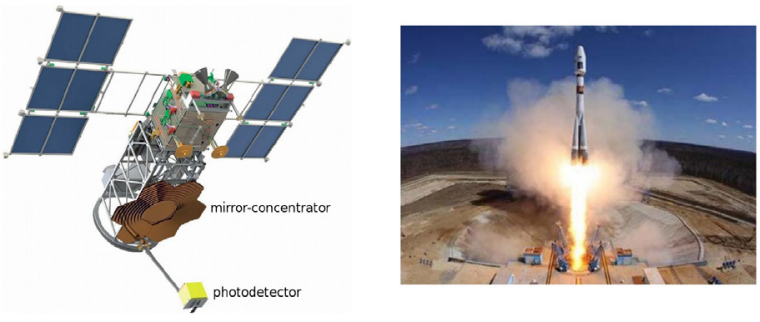

Figure 1. Left: scheme of the TUS detector on-board the "Lomonosov" satellite. Right: the launch of the satellite on April 28,2016

and sensitivity of the PMTs to avoid their saturation when the intensity of the background radiation changes.

The TUS detector consists of two main parts: a modular Fresnel mirror-concentrator and a photo-receiver matrix with the corresponding DAQ electronics (field of view $(\mathrm{FoV}) \pm 4.5^{\circ}$, number of pixels $16 \times 16$ and light guides) (Fig. 1 left). One pixel is a Hamamatsu R1463 PMT with a 13-mm-diameter tube and UV glass window. A PMT quantum efficiency is $\sim 20 \%$ for a wavelength of $350 \mathrm{~nm}$.

TUS has a two-level trigger. The first-level trigger is a threshold trigger: the photodetector modules on board calculates a moving sum of PMT signals during 16 time steps in each channel and looks for a moving sum value above a threshold level. The second-level trigger is a pixelmapping trigger. This procedure selects cases of sequential triggering of spatially contiguous active pixels that are also adjacent in time, allowing for the selection of events with a special spatial-temporal pattern. The design of the TUS detector and its performance is presented in more detail in [5]. 

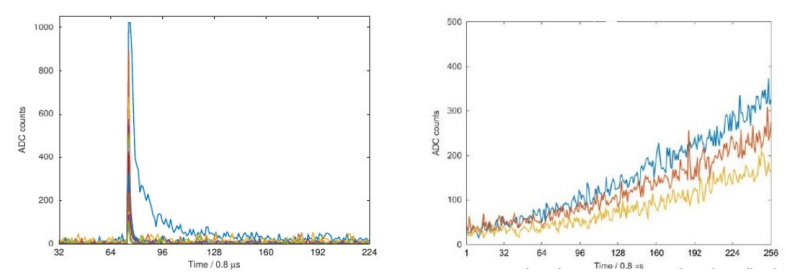

Figure 2. Left: Instant track-like flashes' waveforms of ten PMTs with the highest amplitudes. Right: the waveforms of slow-flashes

\section{Background investigation}

The TUS electronics can operate in four modes intended for detecting various fast optical phenomena in the atmosphere on different time scales.

During two years of operation, the TUS detector has measured about 200,000 events.

More than $80 \%$ of events registered by TUS have noise-like waveforms. The TUS detector has measured numerous UV transient flashes in the EAS mode with different temporal dynamics and spatial structure.

The interesting group of events, averaging about $10 \%$ of the total data set are intense flashes that produced linear tracks across the focal surface (Fig. 2 (left)). Preliminary simulations have shown that protons with energies from $100 \mathrm{MeV}$ up to a few GeV that hit the UV filters approximately parallel to their plane can produce fluorescence and Cherenkov radiation and result in tracks similar to those observed by [6].

Another group of events consists of flashes with amplitudes constantly increasing for more or about $100 \mu \mathrm{s}$, modulated by random fluctuations of the signal, the so-called "slow flashes" (Fig. 2 (right)) Such a flash usually develops simultaneously on the majority of pixels, producing an almost uniform illumination of the focal plane. In most cases, amplitudes continue to increase until the recording is complete. More information about the different types of background events may be found in [7].

\section{EAS candidates}

A few EAS candidates were found and analyzed according to the understanding of EAS physics and the TUS detector simulation. Some details of the analysis criteria may be found in a published paper [8]. Analysis of TUS data shows that most of the events cannot be EAS candidates. They look like a short (about $150 \mu$ s) flash of light. Sometimes such events look like tracks of a few pixels length. The zenith angles of such events are near zero. It means that we have a non-moving flash source of light. There were no Cherenkov flashes at the ends of cascade curves as may be expected in some EAS events. Besides the time durations of the signals are longer than it should be for vertical EASs. The pseudo EAS events distribution on the PMT matrix is homogeneous that excludes the apparatus nature for these events. The majority of EAS-like events can be related to fast anthropogenic signals.

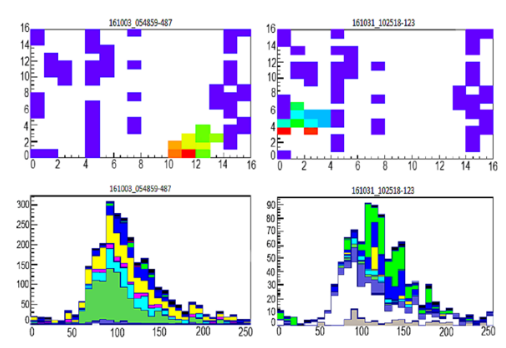

Figure 3. The EAS candidates. Upper plots - image of event with hit pixels and not-working (blue) ones. Bottom plots - the amplitude variation of time for selected hit pixels.

The results of the research of the best and the most convincing EAS candidates are presented in Fig. 3. A summary of the events is shown in Fig. 3: the date and UTC time of the event measurement are the figure title, hit pixels together with dead ones in the upper panel and the amplitude variation of time for selected hit pixels in the lower panel. The markers and colors of the selected pixels on the upper panel indicate the arrival times for the maximum EAS signals in the time steps. Active pixels are grouped in an oblong spot. It can be seen from the waveforms that the characteristic duration of the signal is 70-100 $\mu$ s which is more that one can expect from a vertical EAS. The moment of the maximum of the signal in each pixel has some shift from one pixel to another. This is an argument in favour of a horizontal EAS origin for these events. An important remark about EAS events in Fig. 3 is a temporal sequence of hit pixels which corresponds to moving EAS signal to a moving EAS signal to the outside of the PMT matrix which is a possible reason of the absence of the Cherenkov peak in the bottom plots.

It is important to note that a possible thunderstorm activity was studied in the region of these events measurements. During the time period of the TUS events, no lightning strikes were registered in these regions. This provides a strong support for a non-thunderstorm origin of the events.

Another opportunity for EAS candidate analysis is the reconstruction of their arrival directions. The angles of the found EAS candidates are $\theta=\sim 30^{\circ}, \phi=\sim 225^{\circ}$ for event $\# 487$ and $\theta=\sim 22^{\circ}, \phi=\sim 253^{\circ}$ for event \#123 in the TUS coordinate system.

\section{Calibration}

During the first days of operation several PMTs were broken due to HV tuning system failure. For the same reason, the properties of the remaining PMTs are changed.

Calibration of PMT gains was done based on analyzing background data. For this purpose all time sequences (256 time steps) in all pixels (224 working pixels) of all received events (279662) were classified into 2 categories: pure background pixels and a possible signal of any origin. Classification was done by applying multiple cuts on the data of each time sequence, most importantly a cut on kurtosis, a cut on the slope of the average and cuts on anomalously high and anomalously low mean. Division of data 


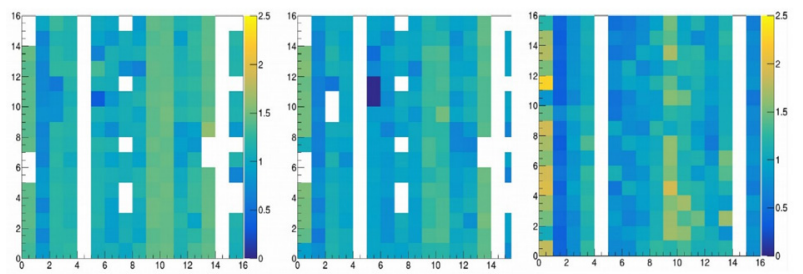

Figure 4. Calibration of PMTs and comparison to pre-flight measurements. Relative PMT gain calibration was done by analyzing background events and is presented here for 2 statistically independent subsets of data (left and center). Pre-flight measurements (right) are shown with 2 PMT modules that later stopped working turned off for comparison

into 3 fully statistically independent samples, corresponding to 6 months of operation each, provide consistently repeating coefficients that validate this assumption. Calibration of PMTs for 2 statistically independent and comparison to pre-flight measurements are shown in fig 4.

The most significant expected shortcoming of this method is that pixels with extremely high or extremely low PMT gains might get in at the edge of some of the statistical cuts and be counted as more moderate than they really are. This may lead to marking several additional pixels as unreliable (pixels with very low gains were already not considered to be reliable anyway) and their exclusion from event reconstruction. The relative PMT calibration was taking into account at the arrival angle evaluation for EAS candidates.

\section{Conclusion}

The TUS detector is operating on board the "Lomonosov" satellite. TUS proved the possibility of registration of UHECRs from the space orbit. During the search for an UHECR EAS a large number of events of various origins that took place in the atmosphere of the Earth were observed. These events may contain some genuine EAS events at energy $>70 \mathrm{EeV}$ which is a lower limit of the sensitivity according to TUS detector simulation [9]. At least two EAS candidates were selected and have been analyzed. A more detailed analysis of this and other EAS candidate events, which were found in the TUS detector data, is in progress.

\section{Acknowledgments}

The TUS experiment on board the "Lomonosov" satellite was realized within the Federal Space Program of Russia with funding by the Russian Space Agency. The data analysis is supported by RFBR grants No. 16-29-13065 and No. 15-02-05498.

\section{References}

[1] Y. Tsunesada, Proc. 33rd Int. Cosmic Ray Conf. Rio de Janeiro (2013)

[2] R.U. Abbasi et al., Astroparticle Physics V64, 49-62 (2015)

[3] RG. K. Garipov et al., Bulletin of the Russian Academy of Sciences: Physics, V79 Issue 3, 326-328, (2015)

[4] The JEM-EUSO Collaboration, Experimental Astronomy, V40 Issue 1, 19-44, (2015)

[5] P. A. Klimov et al., Space Science Reviews, V212 Issue 3-4, 1687-1703 (2017)

[6] Klimov P. A. et al., Bull Russ. Acad. Sci. Phys. 81(4), 407-409 (2017)

[7] B.A. Khrenov at al., Journal of Cosmology and Astroparticle Physics V2017 Issue 09, 006 (2017)

[8] S.V. Biktemerova et al., arXiv:1706.05369v1 [astroph.IM] 16 Jun 2017

[9] A. Grinyuk et al., Astroparticle Physics V90 93-97 (2017) 